\title{
Fuentes antropogénicas y naturales de contaminación atmosférica: estado del arte de su impacto en la calidad fisicoquímica del agua de lluvia y de niebla
}

\author{
Natural and anthropogenic sources of \\ atmospheric pollution: State of the art \\ of its impact on the physicochemical \\ properties of rain water and fog water
}

José Carlos Mora-Barrantes ${ }^{1}$, José Pablo Sibaja- Brenes ${ }^{2}$, Henry Borbón- Alpizar ${ }^{3}$

Mora-Barrantes, J. C; Sibaja- Brenes, J. P;

Borbón- Alpizar, $\mathrm{H}$. Fuentes antropogénicas y naturales de contaminación atmosférica: estado del arte de su impacto en la calidad fisicoquímica del agua de lluvia y de niebla. Tecnología en Marcha. Vol. 34-1. Enero-Marzo 2021. Pág 92-103.

doi https://doi.org/10.18845/tm.v34i1.4806

Escuela de Química. Universidad Nacional, Heredia, Costa Rica. Correo electrónico: jose.mora.barrantes@una.cr (iD https://orcid.org/0000-0002-0409-5276

2 Escuela de Química. Universidad Nacional, Heredia, Costa Rica. Correo electrónico: jose.sibaja.brenes@una.cr

3 Escuela de Química. Universidad Nacional, Heredia, Costa Rica. Correo electrónico: henry.borbon.alpizar@una.cr 


\title{
Palabras claves
}

Costa Rica; agua de lluvia; agua de niebla; contaminantes; atmosfera; fuentes naturales y antropogénicas.

\section{Resumen}

En este artículo se describe el estado del arte en relación con la influencia de las fuentes naturales y antropogénicas de los contaminantes atmosféricos en las características fisicoquímicas del agua de niebla y de lluvia en zonas boscosas. Se recopilaron resultados de investigaciones que se considera facilitarán a la comunidad científica la generación de proyectos en zonas boscosas montañosas. Se determinó que la mayor parte de estudios se han enfocado en el impacto de las emisiones gaseosas de fuentes antropogénicas de zonas urbanas en las características del agua de lluvia. Existen también otras investigaciones sobre la afectación del agua de niebla y de lluvia de zonas boscosas; estas igualmente indican que existe una importante relación de la calidad del agua de niebla y de lluvia de los bosques con las emisiones gaseosas antropogénicas, principalmente. En el caso particular de Costa Rica se han realizado dos pesquisas preliminares respecto al tema. La revisión del estado del arte sugiere un mayor control y seguimiento de los parámetros fisicoquímicos del agua (lluvia y niebla), con mayores tiempos de muestreo y análisis, y la identificación de las fuentes naturales y humanas de los contaminantes en las cercanías de las áreas de estudio.

\section{Keywords}

Costa Rica; water; rain; fog; pollutants; atmosphere; natural and anthropogenic sources.

\begin{abstract}
In this article, a description is presented on the state of the art related with the influence of natural and anthropogenic atmospheric pollutant sources on the physical and chemical properties of the rain water and fog water. It was determined that most of the researches done have been located on urban areas and have evaluated the impact of the anthropogenic emissions to the atmosphere on water quality. There have been a limited quantity of investigations in forest zones. The few ones point out an important influence of human pollutant emissions on the properties of rain water and fog water. In the particular case of Costa Rica, two prelaminar researches have been done. The literature review on the state of the art suggested the need for longer research periods in sampling and analyzing rainwater and fog water, in order to do a better control of their physicochemical parameters. Future researches shall study deeply the natural and anthropogenic sources of atmospheric pollutants in the surroundings of the areas of study.
\end{abstract}

\section{Introducción}

El cambio climático es uno de los principales problemas que afectan a la humanidad, producto de sus efectos medioambientales y, fundamentalmente, debido a su principal acción determinante, como lo es el incremento de los gases de efecto invernadero, resultantes principalmente de las actividades antropogénicas El clima, de forma general, es el resultado de la interacción de la radiación solar, la órbita terrestre, la latitud, la composición atmosférica (temperatura, presión, vientos, precipitaciones), las corrientes oceánicas, el efecto albedo y, sobre todo, los efectos antropogénicos. La aceleración del cambio climático en tiempos recientes ha sido fuertemente influenciada por las actividades humanas, como producto del consumo de combustibles fósiles, resultado en exponencial incremento de gases de efecto invernadero como dióxidos de 
azufre, ozono, metano, dióxido de carbono y dióxido de nitrógeno, entre otros. El aumento de la temperatura global generada por el efecto invernadero es responsable del incremento del nivel del mar, de la disminución de las capas de nieve y hielo, además de la modificación en el estado natural de las precipitaciones. Todo esto impacta los sistemas naturales relacionados al hielo, a los sistemas hidrológicos y a la calidad de las aguas, a los sistemas biológicos marinos y de agua dulce y a la productividad agrícola y forestal.

Producto de lo anterior, el presente trabajo de investigación tiene como objetivo principal el realizar una revisión del estado del arte de la influencia de fuentes naturales y antropogénicas en la composición fisicoquímica de la calidad agua de lluvia y de niebla. El alcance del proyecto contempla el análisis del agua de lluvia y niebla principalmente en zonas montañosas. Se pretende que la investigación desarrollada sirva como insumo para la sensibilización de la población y en determinación preliminar de potenciales fuentes naturales y antropogénicas de contaminación en el agua de lluvia y niebla. Se revisaron diferentes artículos de investigación de bases de datos de editoriales relacionados con cambio climático, principalmente vinculadas al tema de contaminación atmosférica y su impacto sobre el recurso hídrico.

\section{La atmósfera}

La atmosfera es la envoltura gaseosa que rodea totalmente la Tierra; esta se puede dividir en capas concéntricas perfectamente diferenciadas respecto a su composición y densidad. En los $30 \mathrm{~km}$ más próximos a la superficie terrestre está concentrada aproximadamente el 99\% de su composición. Las capas que componen la atmosfera son la troposfera, la estratosfera, la mesosfera, la ionosfera y la exosfera. De estas capas, la troposfera es la de mayor cercanía a la superficie de la tierra y en la que se produce el tiempo atmosférico. La composición de la troposfera es de 78,00\% de nitrógeno, 21,00\% de oxígeno, 0,90\% de argón, 0,03\% de anhídrido acético y agua en cantidades que varían constantemente (vapor, gotas y cristales de hielo) [1]-[3]. La cantidad de vapor de agua contenida en la atmosfera se conoce como humedad atmosférica. Esta ocupa un pequeño volumen, el cual es menor al $2 \%$ del vapor de agua total, no obstante, la humedad atmosférica es el componente más importante desde el punto de vista climático. La entrada de vapor de agua en el aire atmosférico se realiza mediante los procesos de evaporación de aguas superficiales y del agua de los suelos, así como producto de la transpiración de las plantas. La salida del vapor de agua se lleva a cabo por medio de las precipitaciones líquidas y sólidas. El rocío y la escharcha son dos maneras de condensación del exceso de vapor de agua, sobrepasado el punto de saturación; este exceso es depositado en forma de gotas de agua sobre los objetos fríos produciendo el rocío, la niebla y cristales de hielo comúnmente conocidos como escarcha [1]-[3].

La condensación del vapor de agua se produce de forma habitual sobre partículas de polvo, sales, entre otras, llamadas núcleos de condensación. Estas partículas son higroscópicas y favorecen la condensación, inclusive previamente a la saturación. A las partículas que originan núcleos de cristales de hielo se les conoce como núcleos de sublimación. En el caso particular de la condensación, este fenómeno se puede generar por cualquier proceso atmosférico que produzca enfriamiento, como el ascenso de una masa de aire, la mezcla de masas de aire con diferentes temperaturas y el contacto con superficies más frías. Todos estos procesos generan enfriamiento de la masa de aire, causando la disminución de su punto de saturación y la condensación del vapor de agua sobre los núcleos de condensación[1]-[3].

Al enfriarse la masa de aire se generan los procesos de condensación o congelación, los cuales dan lugar a la aparición de gotas de agua o de pequeños cristales de hielo, los cuales irán creciendo y una vez que almacenen el tamaño suficiente, caerán, dando lugar a la precipitación. 
Se puede hacer referencia a precipitaciones líquidas o sólidas. Las sólidas son la nieve y el granizo. Las líquidas son la llovizna, el chubasco y la lluvia [1]-[3].

En la atmósfera, existe gran cantidad de partículas sobre las cuales las moléculas de vapor de agua tienden a congregarse para transformarse en líquido, generando de esta forma, diminutas gotas de agua. La mayor parte de estas partículas de condensación están compuestas por sustancias químicas como sulfatos y nitratos, producidos en el aire como resultado de la combustión de productos abundantes en azufre y ácido nítrico encontrados de forma permanente en la atmosfera [1]-[3].

\section{Contaminantes atmosféricos}

La concentración de contaminantes en la atmósfera es muy variable; depende de la distribución y ubicación de las fuentes de contaminación, la altura de chimeneas, las condiciones topográficas y, en particular, las cambiantes condiciones climáticas [4]. Entre las principales fuentes de contaminación antropogénica del aire están las plantas de calefacción, las centrales térmicas, las incineradoras de residuos, la industria química, el transporte, la minería a cielo abierto y la generación de energía nuclear [5]-[6]. Su acción dañina ha sido de tal dimensión, que el aumento en el nivel de contaminación del aire en las ciudades ha llegado a afectar las zonas aledañas, incluyendo ecosistemas boscosos. Estudios sobre la exposición a contaminantes atmosféricos además han revelado que los contaminantes en ambientes exteriores e interiores están asociados con efectos adversos agudos en la salud de humanos y con el deterioro de los ecosistemas terrestres (flora y fauna) [6].

La contaminación antropogénica del aire se incorpora principalmente a los elementos del agua de lluvia en la capa debajo de las nubes. En este sentido, la precipitación es un elemento importante para la autolimpieza del aire. Investigaciones han demostrado que después de la Iluvia, o incluso durante esta, el aire contiene significativamente menos contaminantes que antes de la precipitación [4]. El contacto del agua de lluvia con los contaminantes atmosféricos genera, entre otros efectos, el fenómeno llamado lluvia ácida, el cual ha sido altamente identificado por la afectación de la calidad del agua, como producto de la presencia de aniones de ácido sulfúrico $\left(\mathrm{SO}_{4}{ }^{2-}\right)$ y de ácido nítrico $\left(\mathrm{NO}_{3}{ }^{1-}\right)$, principalmente [4]-[7]. Por lo tanto, la acidificación implica la presencia de sulfatos y nitratos, los cuales se forman por oxidación de los dióxidos de azufre $\left(\mathrm{SO}_{2}\right)$ y dióxidos nitrógeno $\left(\mathrm{NO}_{x}\right)$ presentes en las emisiones gaseosas, provenientes principalmente de fuentes antropogénicas. Se estima que las fuentes antropogénicas aportan más del $50 \%$ de las emisiones de $\mathrm{SO}_{2}$ y más del 30\% de las emisiones de $\mathrm{NO}_{x}$ [4], [8].

Por su parte, la deposición atmosférica del nitrógeno desempeña un rol importante en el ciclo del nitrógeno. Un aumento en la carga del nitrógeno atmosférico puede generar una saturación de nitrógeno en los ecosistemas. En centros urbanos la principal fuente antropogénica de compuestos nitrogenados en la precipitación es la quema de combustibles fósiles [4]-[9].

Otro compuesto químico que se debe considerar porque también se relaciona con la contaminación atmosférica es el fósforo, presente en forma de fosfatos en aguas naturales. Uno de los principales problemas ambientales es producto de su aporte al proceso de eutrofización. Las cantidades medias de este elemento en aguas residuales alcanzan valores entre los 9,15 $\mathrm{mg} \cdot \mathrm{L}^{-1}$ y los $30 \mathrm{mg} \cdot \mathrm{L}^{-1}[4]-[10]$.

\section{Agua de lluvia y de niebla}

De acuerdo a la definición oficial de la Organización Meteorológica Mundial (OMM), la lluvia es la precipitación de partículas líquidas de agua de diámetro mayor a 0,5 mm o de gotas inferiores dispersas, las cuales precipitan a una velocidad entre 3 y $4 \mathrm{~m} / \mathrm{s}$ en función de su peso 
y de la influencia del viento. Las gotas son dispersas y dependen de la presión atmosférica, la temperatura y humedad [1]-[3]-[11].

Los principales fenómenos atmosféricos visibles del tiempo meteorológico son las nubes, la Iluvia y la niebla, los cuales representan un paso transitorio e indispensable en el ciclo del agua como la evaporación de la humedad desde la superficie terrestre, su transporte hasta niveles superiores de la atmosfera, la condensación del vapor de agua en masas nubosas y el retorno final de agua a la tierra en forma de precipitaciones de lluvia y niebla, entre otros [1]-[3]-[11].

El agua de lluvia y el agua de niebla cumplen un rol fundamental en la limpieza de la atmósfera, proceso mediante el cual se remueve la mayor cantidad de contaminantes. La composición química del agua de lluvia y de niebla presenta variación de un lugar a otro y de una región respecto a otra, como producto de la influencia de fuentes locales [12]-[13], tanto naturales como antropogénicas [12]-[14]. Por lo tanto, determinar la composición del agua (tanto de lluvia como de niebla) es una variable atmosférica que permite comprender el papel del transporte de los componentes solubles de la atmósfera y la contribución de las diferentes fuentes de contaminantes atmosféricos sobre la calidad del agua [12]-[15]. Los fenómenos de agua de lluvia y de niebla funcionan como un sumidero relevante para la materia en forma de gases y partículas, incluyendo metales trazas en la atmósfera, y por lo tanto, desempeñan un rol importante en el control de las concentraciones de estas especies [12]-[16].

Además de la lluvia, es bien sabido que la niebla y las nubes impactan también la composición atmosférica y química del aire. Tanto la niebla como las nubes pueden incorporar gases y especies articuladas en la atmosfera, pudiendo transformar estas especies en otras a través de la fase acuosa o mediante reacciones heterogéneas [4]. Estas nuevas especies formadas pueden afectar la producción agrícola, ya que debilitan las características del suelo, producto de su acumulación y generan procesos como la acidificación, la alcalinización y la metalización, causando una reducción de sedimentos que afecta la flora y fauna [4].

Las emisiones gaseosas pueden incorporarse a los elementos del agua de lluvia y de niebla en la capa debajo de las nubes, haciendo esto que, la precipitación sea un elemento importante en la autolimpieza del aire. La presencia de contaminantes en el agua de la lluvia y de niebla ha incrementado en tiempos recientes. Si bien tanto la lluvia como la niebla son un fenómeno de destilación natural, su formación involucra inicialmente un proceso de nucleación en partículas de aerosol. Cuando el agua se condensa, interactúa con diversas sustancias del aire, por lo tanto, disuelve parte de estas. A medida que el agua de lluvia desciende, arrastra mayor cantidad de impurezas [15]. En efecto, las primeras aguas de lluvia pueden estar mayormente contaminadas; esta condición disminuye a medida que continúa la lluvia, hasta que el agua baja limpia [15]-[17]. Este proceso global permite limpiar el aire también; es así que, después del proceso de lluvia, el contenido de aerosol (amonio, nitrato y sulfato) se reduce entre el $30 \%$ y el $73 \%$, mientras que el de gases (amonio, dióxido de nitrógeno, dióxido de azufre y cloruro) puede disminuir entre el $24 \%$ y el $63 \%$. La habilidad que tiene el agua de lluvia y de niebla en la captura de contaminantes del aire hace de ella un buen medio para el estudio de la contaminación atmosférica [15].

\section{Impacto ambiental de los contaminantes atmosféricos presentes en el agua de lluvia y de niebla}

El agua de lluvia y de niebla tiene una influencia importante en los sistemas naturales y en la salud humana. En el caso particular del agua de niebla, sus propiedades han sido estudiadas durante mucho tiempo por la comunidad científica mundial [18]-[21]. La deposición de agua de niebla es importante para las regiones forestales, tanto para el crecimiento de la vegetación 
como para mantener el balance hídrico. Además, sistemas de colección de agua de niebla se han utilizado para solventar la escasez de agua, como ha ocurrido en regiones de Sur África [18]-[22]-[23]. No obstante, en días de niebla, esta se carga con sustancias orgánicas y gotas de entre $2 \mu m$ y $8 \mu m$ que pueden ser dañinas para la salud si se inhalan [24]-[25]. Otras investigaciones han estudiado la relación entre la composición iónica del agua de niebla y las condiciones marinas, siendo uno de los principales hallazgos que la contribución o influencia de las condiciones marinas es generalmente poca e, inclusive, insignificante en la composición fisicoquímica de la niebla continental [26].

Varios investigadores señalan que los principales iones inorgánicos presentes en la niebla, incluyendo los iones sulfatos $\left(\mathrm{SO}_{4}{ }^{2-}\right)$, nitratos $\left(\mathrm{NO}_{3}{ }^{1-}\right)$, cloruros $\left(\mathrm{Cl}^{-}\right)$, amonio $\left(\mathrm{NH}_{4}{ }^{+1}\right)$, calcio $\left(\mathrm{Ca}^{2+}\right)$ y ion magnesio $\left(\mathrm{Mg}^{2+}\right)$, son producidos por contaminación antropogénica como la producción industrial, las emisiones de automóviles y la construcción de edificios, entre otras formas [24]-[27]-[28]. Varias pesquisas han demostrado la existencia de una relación directa entre la dinámica microfísica y la química del agua de niebla [29]-[32]. Por su parte, otras investigaciones han demostrado que concentraciones de iones en agua de niebla formada en las laderas de las montañas muestran una relación de dependencia con la concentración atmosférica y las propiedades de la niebla [33].

Diferentes estudios han evidenciado la influencia de los contaminantes atmosféricos sobre el agua de niebla y de lluvia. La concentración de iones en agua de niebla y de lluvia es mayor en áreas rurales [25]-[34] que en áreas costeras [35]. Los nutrientes y contaminantes presentes en la niebla y en el agua de las nubes ejercen una fuerte influencia sobre los ecosistemas [36], especialmente en ecosistemas forestales montañosos, en los cuales la niebla es muy frecuente; por lo tanto, se infiere que el agua de niebla es una fuente importante de deposición de iones [37]-[38].

Un estudio realizado en Loja, Ecuador, en la cordillera oriental de Los Andes respecto a la composición química del agua de niebla y de la lluvia, indicó en el caso particular de las muestras de agua, que los iones predominantes son sulfato e hidrógeno, y además que los iones nitrato y amonio desempeñan un papel menor. La investigación resaltó que existe una diferencia pronunciada entre la composición del agua de lluvia y del agua de niebla. La concentración equivalente de ion total de las muestras de niebla y de lluvia es de $139 \mu \mathrm{eq} \mathrm{L}^{-1} \mathrm{y}$ 1534,4 $\mu \mathrm{eq} \mathrm{L}^{-1}$, respectivamente. La apreciable diferencia se debe a los iones hidrógeno $\left(\mathrm{H}^{+}\right)$, amonio $\left(\mathrm{NH}_{4}{ }^{+}\right)$y nitratos $\left(\mathrm{NO}_{3}{ }^{-}\right)$presentes en el agua de niebla [35].

Los iones $\mathrm{H}^{+}, \mathrm{NH}_{4}{ }^{+}, \mathrm{NO}_{3}{ }^{-}$y $\mathrm{SO}_{4}{ }^{2-}$ son indicadores de contaminación antropogénica. Para el caso particular de los compuestos de nitrógeno presentes en el agua de niebla, se ha encontrado que sus principales fuentes son la quema de biomasa, la industria y el tráfico vehicular. No obstante, se deben considerar muchos otros aspectos [35]-[39].

Las diferencias químicas entre la niebla y el agua de lluvia pueden variar en función del tamaño de las gotitas. Las gotas de lluvia son mucho más grandes que las gotas de niebla y pueden formar soluciones más diluidas que las gotas de niebla. Otra diferencia entre la niebla y el agua de lluvia es el desequilibrio iónico en la niebla, ya que en esta predomina la presencia de los cationes sobre los aniones. Este excedente de cationes puede resultar de la presencia de ácidos orgánicos [35]-[39].

En el caso particular del agua de lluvia, D.Gillete et al. [40] y N. Rastogi et al. [41] indican que esta es un mecanismo muy efectivo para absorber y remover partículas de aerosoles presentes en la atmósfera, gracias a los procesos de arrastre "en la nube" y por "debajo de la nube". También D.Gillete et al. [40], Rastogi et al. [41] y Niu et al. [42]. indican que las características del suelo de una determinada región tienen una alta influencia e impacto sobre el pH y la composición química del agua de lluvia. 
Yao W et al.[43] realizaron una investigación durante el 2016 para determinar la composición química del agua de lluvia en tres sitios rurales de la provincia de Hebei, en el norte de China. El propósito principal del estudio fue obtener una comprensión inicial de la química del agua de Iluvia, así como identificar las posibles fuentes que contribuyen en su composición y finalmente, explorar la influencia de fuentes terrestres sobre la composición iónica en zonas no urbanas del norte de China. Entre los resultados están: a) que existe una limitada concentración de aniones en el agua de lluvia (según Migliavacca et al. [44] y Xu et al. [45], esto se debe a la poca presencia de $\mathrm{HCO}_{3}^{-}$y de ácidos orgánicos en el agua de lluvia); b) que el catión calcio fue en el estudio el de mayor abundancia, seguido por el ion amonio, c) que los aniones predominantes son el sulfato y el nitrato (estos datos coinciden con otros estudios realizados por Xu \& Han [45], Huang et al. [46] y Larssen et al. [47]) y d) que se detectaron además altas concentraciones del ion $\mathrm{F}^{-}$. Según lo indican Larssen et al. [47], las concentraciones de $\mathrm{F}^{-}$se pueden deber a la combustión del carbón con alto contenido de fluoruros.

Particularmente en sistemas montañosos y costeros, La Bastille and Pool [48] y los autores Liu et al. [49] indican que la deposición de agua de niebla y de nube sobre la vegetación es una vía de absorción de químicos y de agua contaminantes atmosféricos cerca de la superficie terrestre [50]. Un gran número de estudios han mostrado una alta concentración de moléculas orgánicas e inorgánicas en el agua de niebla [50]-[52].

\section{Afectación de las zonas boscosas por los contaminantes atmosféricos}

La entrada de sustancias químicas provenientes de la niebla y de las nubes en los ecosistemas boscosos es suficientemente significativa como para causar un impacto negativo en el crecimiento del bosque [50]-[53]-[54]. No obstante, la mayoría de las investigaciones sobre el agua de lluvia como fuente de deposición química han sido mayormente desarrollada en áreas con alta frecuencia de nubosidad, en las cuales se espera que reciban masas de aire contaminadas, particularmente en regiones templadas como Norte América y Europa [50]-[55][58].

Una investigación de Wen et al. [50] realizada en el año 2005 cuantificó y reportó la concentración iónica en agua de niebla de un bosque tropical. El estudio indicó valores altos de pH tanto en el agua de niebla como agua de lluvia; estos datos se pueden atribuir a las altas concentraciones de iones amonio, calcio y magnesio, comparadas con las bajas concentraciones de sulfatos y nitratos [50]. En general, el ion amonio es mayormente atribuido a la producción bacteriana derivada de actividades de agricultura, y se estima que este ion neutraliza la acidez del agua de la nube [58]. En el estudio, Wen et al. [50] reportaron el ion $\mathrm{HCO}_{3}^{-1}$ como el de mayor concentración. Estudios previos habían reportado también el ion $\mathrm{HCO}_{3}^{-1} \mathrm{Como}^{-1}$ uno de los iones de mayor importancia en nieblas tropicales [59], [60]. El estudio de Wen et al. [50] indica una mayor concentración de iones en el agua de niebla respecto al agua de lluvia. Lo cual coincide de igual manera con otros estudios previos [61].

En Costa Rica se llevó a cabo un estudio preliminar para determinar la composición química del agua de lluvia y de niebla en tres sitios de la Reserva Biológica "Monteverde", en Puntarenas, durante el periodo comprendido entre octubre del 2009 y enero del 2010. Para el agua de lluvia la cantidad de muestras recolectadas fueron diez y para el agua de niebla cinco. El estudio indicó concentraciones bajas de in hidronio $\left(\mathrm{H}_{3} \mathrm{O}^{+}\right)$tanto en el agua de lluvia como de niebla; estas concentraciones se pueden atribuir a una importante presencia de sales de calcio, en comparación con las bajas concentraciones de $\mathrm{NO}_{3}^{-}$y $\mathrm{SO}_{4}{ }^{2-}$. Se concluye además que las concentraciones reportadas de $\mathrm{Ca}^{2+}, \mathrm{K}^{+}, \mathrm{Mg}^{2+} \mathrm{y} \mathrm{SO}_{4}{ }^{2-}$ en las muestras de agua de lluvia y de niebla se debieron a la influencia del ecosistema marino cercano [62]. 
Otro estudio preliminar desarrollado en Costa Rica (Parque Nacional "Volcán Poás") durante los meses de mayo a octubre del 2017 por el Laboratorio de Química de la Atmósfera, de la Universidad Nacional, analizó en el agua de lluvia parámetros como pH, conductividad eléctrica y aniones $\left(\mathrm{F}^{-}, \mathrm{Cl}^{-}, \mathrm{NO}_{3}^{-}\right.$y $\left.\mathrm{SO}_{4}{ }^{2-}\right)$. Los valores más significativos fueron encontrados cerca del punto denominado foco emisor o el mirador para visitantes fueron $\mathrm{pH}=2,50 ; 15,48 \pm 0,22 \mathrm{mg} / \mathrm{L}$ para F-; 205,2 \pm 3,3 mg/L para $\mathrm{Cl}^{-}$, y 64,0 \pm 1,4 mg/L para el $\mathrm{SO}_{4}{ }^{2-}[63]$.

Vásquez et al. [62] y Bolaños et al. [63] recomiendan el monitoreo constante del agua de lluvia y de niebla, con el fin de contar con mayores datos sobre la influencia de fuentes de contaminación naturales y antropogénicas en ecosistemas naturales costarricenses. Estos autores indican la necesidad de analizar una mayor cantidad de muestras y, por lo tanto, de contaminantes (aniones y cationes), así como de realizar más extensos periodos de muestreo y análisis (mínimo dos años).

Vásquez et al. [62] y Bolaños et al. [63] indican que un mayor control y seguimiento de la composición fisicoquímica del agua de lluvia y de niebla es fundamental para el establecimiento de relaciones entre estas (Iluvia y niebla) y las emisiones antropogénicas y naturales, así como para verificar la influencia de los contaminantes atmosféricos en los patrones de lluvia y sus efectos en el agua de consumo humano y la flora y la fauna de los ecosistemas naturales.

\section{Conclusiones}

La lluvia y el agua de niebla cumplen un rol fundamental en la limpieza de la atmósfera, debido a que mediante este proceso se remueve la mayor cantidad de contaminantes. La composición química del agua de lluvia y de niebla varía de un lugar a otro, esto como producto de la influencia de fuentes locales de emisiones tanto naturales como antropogénicas.

La contaminación antropogénica del aire se incorpora principalmente a los elementos del agua de lluvia en la capa debajo de la nube, por lo que la precipitación es un elemento importante en la autolimpieza del aire. Algunas investigaciones han demostrado que después de la lluvia o incluso durante esta, el aire contiene significativamente menos contaminantes previos a la precipitación.

La mayoría de las investigaciones en el mundo se han enfocado en el impacto ocasionado por las fuentes antropogénicas de contaminantes atmosféricos generados en zonas urbanas sobre las propiedades fisicoquímicas del agua de lluvia.

Las investigaciones sobre la influencia de contaminantes atmosféricos sobre la composición iónica del agua de lluvia y de niebla de zonas boscosas ha sido limitada; no obstante, ha aumentado en los últimos años. Los principales estudios se han realizado en zonas montañosas relativamente cercanas a áreas urbanas.

Pesquisas desarrolladas en bosques naturales han determinado que los iones $\mathrm{H}^{+}, \mathrm{NH}_{4}{ }^{+}, \mathrm{NO}_{3}{ }^{-}$y $\mathrm{SO}_{4}{ }^{2-}$ son indicadores de los efectos nocivos de la contaminación antropogénica, producto de la quema de biomasa, la industria y el tráfico vehicular.

En el caso particular de Costa Rica se han realizado dos pesquisas preliminares respecto al tema, una de estas, en la Reserva Biológica "Monteverde" y otra en el Parque Nacional "Volcán Poás". Para el primer estudio se evidenciaron concentraciones bajas de $\mathrm{H}_{3} \mathrm{O}^{+}$tanto en el agua de lluvia como en la de niebla. En el Volcán Poas los valores más significativos de concentración de iones hidronio y aniones fueron encontrados cerca del foco emisor o mirador para .

Se recomienda un mayor control y seguimiento de los parámetros fisicoquímicos del agua de Iluvia y de niebla, con mayores tiempos de muestreo y análisis, además de la identificación de 
las fuentes naturales y humanas de emisión de contaminantes atmosféricos en las cercanías de las áreas de estudio, en este caso particular, los bosques naturales montañosos.

Se recomienda además el desarrollo de proyectos de investigación en los bosques que contemplen de forma más integral los siguientes aspectos: a) el estudio de la variación fisicoquímica de la calidad del aire, b) el análisis de la variación fisicoquímica del agua de niebla y de lluvia, d) la determinación del impacto ambiental en la reservas de bosques o parques nacionales producto de las emisiones atmosféricas de fuentes naturales y antropogénicas mediante el uso del modelo de dispersión de contaminantes y e) el desarrollo de procesos de capacitación y sensibilización con la población local y los trabajadores sobre los peligros, riesgos y medidas de prevención asociados con la variabilidad de la calidad del aire y del agua en los sitios de estudio.

\section{Referencias}

[1] M. Andrades y C. Múñez, "Fundamentos de climatología", en Material Didáctico: Agricultura y Alimentación, Universidad de la Rioja. Servicio de Publicaciones. 2012 [En línea]. Disponible en: https://archive.org/stream L2013FundamentosDeClimatologia/2013_fundamentos-de-climatologia_djvu.txt. [Accesado: 22 dejul, 2019]

[2] J. Barrio, B. Anton, y D. Andrés, Física y química, 3. ${ }^{a}$ ed.. Madrid: Editorial Editex, 2008.

[3] L. Ayala, "Determinación de las concentraciones de iones presentes en el agua de lluvia de la zona urbana de la ciudad de Cuenca, causantes de lluvia ácida", tesis doctoral, Universidad Politécnica Salesiana, Ecuador, 2014.

[4] J. Pokrývková, L. Lackóová, J. Fuska, L.Tátošová, and A. Policht-Latawiec, " The impact of air pollution on rainwater quality," Rocznik Ochrona Srodowiska, vol. 18, no. 1, pp. 303-231, 2016.

[5] A. Sanmi, "Effect of air pollution on rain water: A case study of Ado-Ekiti, Nigeria. International," Journal of Advanced Engineering Research and Science (IJAERS), vol. 5,no. 8, pp. 19-24, 2018. doi: 10.22161/ ijaers.5.8.3

[6] A. Mishra, K.A. Singh, P. Pandey, S. Yadav, A.H. Khan, and S.C. Barman, "Urban air pollution and their effects on rain water characteristics in Lucknow City," India Journal of Environmental Research and Development, vol. 6 , no. 4, pp. 1127-1132, 2012.

[7] S.A. Iqbal, and Y. Mido, Chemistry of Air and Air Pollution. New Delhi: Discovery Publishing House, 2010.

[8] G. Schwedt, The essential guide to environmental chemistry. New York, USA: John Wiley and Sons, 2001.

[9] P. Brimblecombe, H. Hara, D. Houle, and M. Novak, Acid Rain-Deposition to Recovery. Dordrech, Netherland: Springert, 2007.

[10] M. Radojević, and V.N. Bashkin, Practical Environmental Analysis. Cambridge: Royal Society of Chemistry, 2006.

[11] Organización Meteorológica Mundial, “Atlas de Nubes”, London: Organización Mundial de la Salud, 1987.

[12] A. Doria, "Metales pesados ( $\mathrm{Cd}, \mathrm{Cu}, \mathrm{V}, \mathrm{Pb})$ en agua lluvia de la zona de mayor influencia de la mina de carbón en La Guajira, Colombia", Revista Colombiana de Química, vol. 46, no. 2, pp. 37-44, 2017.

[13] J. Seinfeld, and S. Pandis, Atmospheric Chemistry and Physics. From air pollution to climate change. USA: Wiley - Interscience, 2006.

[14] E. Ramirez, R. Miranda, Y. Garcia, I. Balderas, H. Bravo, R. Sosa, P. Sánchez, A. Alarcon, A, M. Torres, and J. Kahl, "Chemical composition of rainwater in northeastern México," Atmósfera, vol. 23, no. 3, pp. 213-224, 2010.

[15] L. Khemani, T. Momin, M. Naik, R. Prakasa, R. Kumar, and M. Ramana, M, "Impact of alkaline particulates on pH of rainwater in India," Water Air Soil Pollution, vol. 25, no. 4, pp 365-376, 1985.

[16] C. Samara, R. Tsitouridou, and Ch. Balafoutis, "Chemical composition of rain in Thessaloniki, Greece, in relation to meteorological conditions," Atmospheric Environment. Part B, Urban Atmosphere, vol. 26, no. 3, pp. 359-367, 1992. doi: 10.1016/0957-1272(92)90011-G

[17] M. Romeo, and D. Nenita, "Rainwater chemistry and biochemical effects: Basis for air quality assessment of Cagayan de Oro city, Philippines," Mindanao Journal of Science and Technology, vol. 9, pp. 29-72, 2011. 
[18] Y. Yanyu, N. Shengj, Z. Lijuan, Z. Yu, and X. Feng, "The influences of macro- and microhphysical characteristics of sea-fog on fog-water chemical composition," Advances in Atmospheric Sciences, vol. 3, no. 3, pp. 624-636, 2014. doi: 10.1007/s00376-013-3059-2

[19] S. Fuzzi, J. Castillo, E. Jiusto, and G. Lala, "Chemical composition of radiation fog water at Albany, New York, and its relationship to fog microphysics", Journal of Geophysical Research, vol. 89, no. D5, pp. 7159-7164, 1984. doi: 10.1029/JD089iD05p07159

[20] S.X. Fan, H.L. Huang, K.H. Gu, H.S. Li, B. Zhu, H.L. Zhang, and Y. Fan, "Effect of Fog Process on the Size Distribution of Polycyclic Aromatic Hydrocarbons in the Atmospheric Aerosol PM10. Gaodeng Xuexiao Huaxue Xuebao / Chemical Journal of Chinese Universities, vol. 31, no. 12, pp. 2375-2382, 2010. [On line]. Available: https://www.researchgate.net/publication/283957537 Effect of Fog Process on the Size Distribution of Polycyclic Aromatic Hydrocarbons in the Atmospheric Aerosol PM10. [Accessed: Jul 14, 2019]

[21] E.Strater, A. Westbeld, and O. Klemm, "Pollution in coastal fog at Alto Patache, Northern Chile", Environmental Science and Pollution Research, vol. 17, no. 9, pp. 1563-1573, 2010. doi:10.1007/s11356-010-0343-x

[22] T. Wrzesinsky, and O. Klemm, "Summertime fog chemistry at a mountainous site in central Europe", Atmospheric Environment, vol. 34, no. 9, pp. 1487-1496, 2000. doi: 10.1016/S1352-2310(99)00348-9

[23] M.V.M Jaén, "Fog water collection in a rural park in the Canary Islands (Spain)," Atmospheric Research, vol. 64, no. 1, pp. 239-250, 2002. doi:10.1016/S0169-8095(02)00095-9

[24] M. Millet, A. Sanusi, and H. Wortham, "Chemical composition of fogwater in an urban area: Strasbourg (France)", Environmental Pollution, vol. 94, no. 3, pp. 345-354, 1996. doi: 10.1016/S0269-7491(96)00064-4

[25] S. Fan, X. Yang, T. Fan, and F. Han, "Metal elements and water-soluble anion concentrations in winter fog water in Nanjing," Huanjing Kexue Xuebao / Acta Scientiae Circumstantiae, vol. 29, no. 9, pp. 1878-1885, 2009. [On line]. Available: https://www.researchgate.net/publication/287953233 Metal elements and watersoluble anion concentrations in winter fog water in Nanjing. [Accessed: March 14. 2019]

[26] C. Lu, S. Niu, L. Tang, J. Lv, L. Zhao, and B. Zhu,B, "Chemical composition of fog water in Nanjing area of China and its related fog microphysics", Atmospheric Research, vol. 97, no. 1-2, pp 47-69, 2010. doi: 10.1016/i.atmosres.2010.03.007

[27] K. Ali, G. Momin, S. Tiwari, P. Safai, D. Chate, and P. Rao, P, "Fog and precipitation chemistry at Delhi, North India", Atmospheric Environment, vol. 38, no. 25, pp. 4215-4222, 2004. doi: 10.1016/j.atmosenv.2004.02.055

[28] M. Błas, Z. Polkowska, M. Sobik, K. Klimaszewska, K. Nowinski, and J. Namiesnik, "Fog water chemical composition in different geographic regions of Poland," Atmospheric Research, vol. 95, no. 4, pp. 455-469, 2010. doi: 10.1016/j.atmosres.2009.11.008

[29] B. Arends, G. Kos, R. Maser, D. Shell, W. Wobrock, P. Winkler, J. Ogren, K. Noone, A. Hallberg, B. Svenningsson, A. Wiedensohler, H. Hansson, A. Berner, I. Solly, and C. Kruisz, "Microphysics of clouds at Kleiner Feldberg," Journal of Atmospheric Chemistry, vol. 19, no. 2, pp. 59-85, 1994. doi: 10.1007/BF00696583

[30] S.Fuzzi, M. Facchini, G. Orsi, G. Bonforte, W. Martinotti, G. Ziliani, P. Mazzalit, M. Grosa, E. Rampado, P. Vitali, R. Raffaeelli, A. Gianpaolo, and S. Grotti, " The NEVALPA project: A regional network for fog chemical climatology over the Po Valley basin," Atmospheric Environment, vol. 30, no. 2, pp. 201-213, 1996. doi: $10.1016 / 1352-2310(95) 00298-D$

[31] D. Moller, K. Acker, and W. Wieprecht, "A relationship between liquid water content and chemical composition in clouds," Atmospheric Research, vol. 41, no. 3, pp. 321-335., 1996. doi: 10.1016/0169-8095(96)00017-8

[32] W. Elbert, M. Hoffmann, M. Kramer, G. Schmitt, and M. Andreae, "Control of solute concentrations in cloud and fog water by liquid water content," Atmospheric Environment, vol. 34, no. 7, pp. 1109-1122, 2000. doi:10.1016/S1352-2310(99)00351-9

[33] M.Y. Igawa, T. Tsutsumi, T. Mori, and H. Okochi, "Fogwater chemistry at a mountainside forest and the estimation of the air pollutant deposition via fog droplets based on the atmospheric quality at the mountain base," Environmental Science \& Technology, vol. 32, no. 11, pp. 1566-1572, 1998. doi:10.1021/es970213x

[34] B. Bao, J. Shu, and B. Zhu, "Study on physicochemical properties of urban fog in Shanghai," Journal of Nanjing Institute of Meteorology, vol. 18, no. 1, pp. 114-118, 1995. [On line]. Available: http://en.cnki.com.cn/ Article en/CJFDTotal-NJQX501.016.htm. [Accessed: May 19. 2019]

[35] E. Beiderwieden, T.Wrzesinsky, and O. Klemm, "Chemical characterization of fog and rain water collected at the eastern Andes cordillera," Hydrology and Earth System Sciences, vol. 9, no. 3, pp. 185-19, 2005. [On line]. Available: https://hal.archives-ouvertes.fr/hal-00304817/document. [Accessed: May 19. 2019]

[36] J. Fisak, M. Tesar, D. Rezacova, V. Elias, V. Weignerova, and D. Fottova, "Pollutant concentrations in fog and low cloud water at selected sites of the Czech Republic," Atmospheric Research, vol. 64, no. 1-4, pp. 75-87, 2002. doi:10.1016/S0169-8095(02)00081-9 
[37] C. Gordon, R. Herrera, and T. Hutchinson, "Studies of fog events at two cloud forests $20 \mathrm{~km}$ near Caracas, Venezuela: II. Chemistry of fog," Atmospheric Environment, vol. 28, no. 2, pp. 323-337, 1994. doi:10.1016/13522310(94)90108-2

[38] J. Walmsley, R. Schemenauer, and H. Bridgman, "A method for estimating the hydrologic input from fog in mountainous terrain," Journal of Applied Meteorology, vol. 35, pp. 2237-2249. doi:10.1175/15200450(1996)035<2237:AMFETH>2.0.CO;2

[39] M. Keller, D. Jacob, S. Wofsy, and R. Harris, "Effects of tropical deforestation on global and regional atmospheric chemistry," Climate Change, vol. 19, no. 1-2, pp. 139-158., 1991. [On line].: doi:10.1007/BF00142221, [Accessed: May 19, 2019]

[40] A. Tang, G. Zhuang, Y. Wang, H. Yuan, and Y. Sun, "The chemistry of precipitation and its relation to aerosol in Beijing," Atmospheric Environment, vol. 39, no. 19, pp. 3397-3406, 2005. "doi: 10.1016/j.atmosenv.2005.02.001"

[41] Z. Xu, Y. Wu, W. Liu, C. Liang, J. Ji, T. Zhao, and X. Zhang, "Chemical composition of rainwater and the acid neutralizing effect at Beijing and Chizhou city, China," Atmospheric Research, vol 164-165, pp. 278-285, 2015. "doi: 10.1016/i.atmosres.2015.05.009"

[40] D. Gillette, and P. Sinclair, "Estimation of suspension of alkaline material by dust devils in the United States," Atmospheric Environment, vol. 24, no. 5, pp. 1135-1142, 1990. doi: 10.1016/0960-1686(90)90078-2

[41] N. Rastogi, and M. Sarin, "Chemical characteristics of individual rain events from a semiarid region in India: Three-year study," Atmospheric Environment, vol. 39, no. 18, pp. 3313-3323, 2005. doi: 10.1016/j.atmosenv.2005.01.053

[42] H.W. Niu, Y.Q. He, X.X. Lu, J. Shen, J.K. Du, T. Zhang, T. Pu, H.J. Xin, and L. Chang, "Characteristics of modern atmospheric dust deposition in snow in the Mt. Yulong region, southeastern Tibetan plateau," Journal of Asian Earth Science, vol. 94, pp. 45-54, 2014. doi: 10.1016/j.jseaes.2014.08.003

[43] W. Yao, X. Zhifang, L. Wenjing, Z. Tong, X. Zhao, X. H. Jiang, H. Y. Chong, Z. Li, and Z. Xiaode, "Chemical compositions of precipitation at three non-urban sites of Hebei Province, north China: Influence of terrestrial sources on ionic composition," Atmospheric Research, vol. 181, pp. 115-123, 2016.

[44] D. Migliavacca, E.C. Teixeira, F. Wiegand, A.C.M. Machado, and J. Sanchez, "Atmospheric precipitation and chemical composition of an urban site, Guaíba hydrographic basin, Brazil," Atmos. Environ, vol. 39, no. 10, pp. 1829-1844, 2005. doi: 10.1016/j.atmosres.2016.06.009

[45] Z. Xu, and G. Han, "Chemical and strontium isotope characterization of rainwater in Beijing, China," Atmospheric Environment, vol. 43, no. 12, pp. 1954-1961, 2005. doi: 10.1016/j.atmosenv.2009.01.010

[46] D.Y. Huang, Y.G. Xu, P. Peng, H.H. Zhang, and J.B. Lan, "Chemical composition and seasonal variation of acid deposition in Guangzhou, South China: Comparison with precipitation in other major Chinese cities," Environmental. Pollution, vol. 157, no. 1, pp. 35-41, 2009. doi: 10.1016/i.envpol.2008.08.001

[47] T.Larssen, A. Semb, J. Mulder, I. Muniz, R. Vogt, E. Lydersen, V. Angell, T. Dagang, O. Eilester, and H.M. Seip, "Acid deposition and its effects in China: An overview," Environmental Science \& Policy, vol. 2, no. 1, pp. 9-24, 1999. doi: 10.1016/S1462-9011(98)00043-4

[48] A. LaBastille, and D.J. Pool, "On the need for a system of cloud forests parks in Middle-America and Caribbean," Environmental Conservation, vol. 5, no. 3, pp. 183-190, 1978. doi: $\underline{10.1017 / \text { S0376892900005890 }}$

[49] W.Liu, F.R. Meng, Y.P. Zhang, Y.H. Liu, and H.M. Li, " Water input from fog drip in the tropical seasonal rain forest of Xishuangbanna, southwest China," Journal of Tropical Ecology, vol. 20, no. 5, pp. 417-424, 2004. doi: $10.1017 / S 0266467404001890$.

[50] J. Wen, P. Yi, M. Hong, F. Meng, H. Yu, and M. Chang, "Fog and Rainwater Chemistry in the Tropical Seasonal Rain Forest of Xishuangbanna, southwest China," Water, Air, and Soil Pollution, vol. 167, no. 1-4, pp. 295-309, 2005. [On line]. Available: https://link.springer.com/article/10.1007/s11270-005-0080-9, [Accessed: May 20, 2019]

[51] P. Herckes, L. Trenary, M.P. Hannigan, T. Lee, and Jr.J..L. Collett, in Cloud and fog processing of atmospheric organic compounds, R. S. Schemenauer and H. Puxbaum, Eds. Canada, 2001.

[52] F. Joos, and U. Baltensperger, "A field study on chemistry S(IV) oxidation rates and vertical transport during fog conditions," Atmospheric Environment, vol. 25, no. 2, pp. 217-230, 1991. doi: 10.1016/0960-1686(91)90292-F

[53] R.M. Cox, J. Spavold-Tims, and R.N. Hughes, "Acid fog and ozone: Their possible role in birch deterioration around the Bay of Fundy, Canada," Water Air Soil Pollution, vol. 48, no. 1-2, pp. 263-276, 1989. [On line ]. Available: https://link.springer.com/article/10.1007/BF00282383, [Accessed: March 14, 2019] 
[54] M.H. Unsworth, "Evaporation from forests in cloud enhances the effects of acid deposition," Nature International Journal of Science, vol. 312, pp. 262-264, 1984. https://www.nature.com/articles/312262a0

[55] J.Jr. Collett, K.J. Hoag, X. Rao, and S.N. Pandis, "Internal acid buffering in San Joaquin Valley fog drops and its influence on aerosol processing," Atmospheric Environment, vol. 33, no. 29, pp. 4833-4847, 1999. doi: 10.1016/S1352-2310(99)00221-6

[56] G.M. Lovett, "Atmospheric deposition of nutrients and pollutants in North America: An ecological perspective," Ecological Application, vol. 29, no. 4, pp. 629-650, 1994. doi: 10.2307/1941997

[57] R.S. Schemenauer, "Acidic deposition to forests: The 1985 Chemistry of High Elevation Fog (CHEF) project," Atmosphere-Ocean, vol. 24, no. 4, pp. 303-328, 1986. doi: 10.1080/07055900.1986.9649254

[58] V.K. Saxena, and N.H. Lin, "Cloud chemistry measurements and estimates of acidic deposition on an above cloudbase coniferous forest," Atmospheric Environment vol. 24, no. 2, pp. 329-352, 1990. doi: 10.1016/09601686(90)90113-2

[59] E.D. Eckardt, and R.S. Schemenauer, "Fog water chemistry in the Namib Desert, Namibia," Atmospheric Environment, vol. 32, no. 14-15, pp. 2595-2599, 1998. doi: 10.1016/S1352-2310(97)00498-6

[60] R. S. Schemenauer, and P. Cereceda, "Monsoon cloud water chemistry on the Arabian Peninsula," Atmospheric Environment, vol. 26, no. 9, pp. 1583-1587, 1992. doi:10.1016/0960-1686(92)90058-S

[61] J. Jr. Collett, K.J. Hoag, X. Rao, and S.N. Pandis, "Internal acid buffering in San Joaquin Valley fog drops and its influence on aerosol processing," Atmospheric Environment, vol. 33, no. 29, pp. 4833- 4847, 1999. doi: 10.1016/S1352-2310(99)00221-6

[62] T. Vásquez, R. Alfaro, J. Sibaja, G, Esquivel y J. Valdés, "Composición química del agua de lluvia y de niebla recolectada en la Reserva Biológica 'Monteverde'," Uniciencia, vol. 26, no. 1-2 pp. 51-63, 2012. [On line]. Available: https://www.revistas.una.ac.cr/index.php/uniciencia/article/view/3862/3703.[Accessed: Jun 6, 2019 ]

[63] K. Bolaños, J. Sibaja, J.Mora, D. Umaña, M. Cambronero, L. Sandolval y M. Martínez, "Estudio preliminar sobre la composición atmosférica del agua de lluvia en y los alrededores del Parque Nacional del Volcán Poás", en Y. Morales-López (Ed.), Memorias del I Congreso Internacional de Ciencias Exactas y Naturales de la Universidad Nacional, Costa Rica, Universidad Nacional, Heredia, e142, pp. 1-11, 2019. doi: 10.15359/ cicen.1.29 